\title{
Evidence of Spontaneous Post-transplant HCV Eradication in Two Failed DAA Treatments Awaiting Liver Transplantation
}

\author{
Ilaria Lenci ${ }^{1}$ - Alessandra Bosa ${ }^{1} \cdot$ Martina Milana $^{1} \cdot$ Leonardo Baiocchi $^{1}$. \\ Francesco Paolo Antonucci ${ }^{2} \cdot$ Marianna Aragri $^{2} \cdot$ Francesca Ceccherini-Silberstein $^{2}$. \\ Carlo Federico Perno ${ }^{2} \cdot$ Giuseppe Tisone ${ }^{3} \cdot$ Mario Angelico ${ }^{1}$
}

Received: 16 March 2017/ Accepted: 25 April 2017

(C) Springer Science+Business Media New York 2017

Keywords DAA treatment $\cdot \mathrm{HCV}$ eradication $\cdot$ Liver transplantation

\section{Introduction}

Recurrence of $\mathrm{HCV}$ infection (rHCV) in patients with detectable HCV-RNA at the time of liver transplantation (LT) is almost universal and is associated with a rapidly progressing course, leading to chronic hepatitis and cirrhosis [1]. Transplant recipients with high levels of HCVRNA prior to LT are more likely to experience fibrosis progression and poor outcome [1]. It is therefore essential to consider early antiviral therapy in the transplant setting, since achievement of sustained virological response (SVR) improves clinical outcomes and survival rates in LT recipients [2]. The recent introduction of direct-acting antivirals (DAAs) has revolutionized the management of HCV therapy [3]. The use of new DAAs has resulted in high SVR rates, with short treatment courses and excellent safety profiles. Yet, the optimal timing of antiviral therapy in the liver transplant setting is still under discussion.

We report here two cases of LT in patients with endstage $\mathrm{HCV}$-related cirrhosis recently performed at our

Ilaria Lenci

ilaria.lenci@uniroma2.it

1 Hepatology Unit, Department of Experimental Medicine and Surgery, Policlinico Tor Vergata, Rome, Italy

2 Virology Unit, Department of Experimental Medicine and Surgery, Policlinico Tor Vergata, Rome, Italy

3 Liver Transplant Center, Department of Experimental Medicine and Surgery, Policlinico Tor Vergata, Rome, Italy centre, which presented unusual features, which we believe deserve attention.

\section{Case Reports}

The first case was a 53-year-old woman with compensated liver cirrhosis who was listed for OLT because of a hepatocarcinoma (HCC) within the Milan criteria (a single nodule, $3 \mathrm{~cm}$ in diameter). She also had type II diabetes mellitus treated with insulin and clinically significant portal hypertension, with esophageal varices. The viral genotype was $1 \mathrm{~b}$, and there was no evidence of concomitant HBV infection. In the past, the patient had received three courses of interferon-alfa treatment, discontinued due to severe thrombocytopenia. In July 2015, the patient was given ledipasvir and sofosbuvir treatment for 12 weeks, attaining early HCV-RNA undetectability. Yet, she relapsed 3 months after the end of therapy (January 2016), when HCV-RNA levels of $1829 \mathrm{UI} / \mathrm{mL}$ (Abbott RealTime HCV assay, lower limit of detection $<12 \mathrm{IU} / \mathrm{mL}$ ) were detected. By HCV sequencing, the presence of resistant associated substitution (RASs) was documented at this time, in NS5A ( $\mathrm{Y} 93 \mathrm{H}$ associated with resistance to ledipasvir), and in NS5B (L159F and C316 N associated with resistance for Sofosbuvir). The patient underwent liver transplantation 1 month later (February 2016), and serum HCV-RNA at time of surgery was found to be $24 \mathrm{IU} / \mathrm{mL}$. During the surgery, the patient received a transfusion with 2 blood and 10 plasma units, and afterward, there was no need of dialysis. HCV-RNA was also assayed by using the Abbott RealTime HCV assay in the explanted liver (after tissue preservation at $-80{ }^{\circ} \mathrm{C}$ ) and resulted positive with $3.1 \mathrm{IU} /$ ugRNA. After LT, the patient was started on immunosuppressive treatment with tacrolimus and everolimus. 
Serum HCV-RNA was repeatedly assayed after OLT, at 1 , $2,8,16$, and 24 weeks, respectively, and in all cases, it was found to be undetectable, thus showing viral eradication.

The second case was a 46-year-old man with liver cirrhosis due to genotype $3 \mathrm{HCV}$ infection and previous alcohol abuse. He had experienced several episodes of liver decompensation and variceal bleeding, which leads to the insertion of a transjugular intrahepatic portosystemic shunt in 2013. Hepatitis B test results were negative. The patient, who was treatment naïve, was started on sofosbuvir and ribavirin treatment in February 2015, which lasted 24 weeks (at that time, daclatasvir and other DAAs were not yet available in Italy), achieving early HCV-RNA undetectability. Yet, a virological relapse was observed 12 weeks after end of treatment, when an HCV-RNA level of $1024 \mathrm{IU} / \mathrm{mL}$ was detected. No RAVs were documented at this time in all 3 genes tested (NS3, NS5A, and NS5B). Serum viremia was then repeated at 3-month intervals, with values ranged between 200 and $500 \mathrm{IU} / \mathrm{mL}$. The patient underwent liver transplantation in June 2016 (after 28 weeks from end of treatment), and HCV-RNA levels were to be undetectable $(<12 \mathrm{IU} / \mathrm{mL})$ in serum at the time of surgery. Also the HCV-RNA assay performed in the explanted liver resulted undetectable $(<12 \mathrm{IU} / \mathrm{mL})$. During and after the surgery, the patient did not require transfusions or dialysis. After LT, the patient was started on immunosuppressive therapy with tacrolimus and everolimus. HCVRNA was assayed at 4,8 , and 12 weeks after LT, respectively, and in all cases, it was found to be undetectable.

\section{Discussion}

According to previous studies, during the first 2 days after $\mathrm{LT}$, there is a rapid decline of hepatitis $\mathrm{C}$ virions, likely due to the removal of the infected liver, which is the major site of HCV replication [4]. This finding also suggests that the lifetime of serum hepatitis $\mathrm{C}$ virions is remarkably short and that extrahepatic replication poorly contributes to the total virus pool.

Both our patients had received a complete course of DAAs therapy prior to LT. Differently, the majority of patients reported by Curry et al. [5] did not complete a full course of antiviral treatment while in the wait-list and did not achieve undetectable HCV-RNA at time of LT.

Both our patients underwent virological relapse after treatment cessation, but the viral load remained in a rather low range until the time of transplant surgery that was shortly after the discovery of relapse. Thus, it is conceivable that removal of the infected liver was sufficient to avoid reinfection of the new graft in these patients due to the small concentrations of circulating virions, also considering a short anhepatic phase. It is also possible that antiviral therapy produced some alterations in $\mathrm{HCV}$ genome thus influencing its infectiveness. In one case, there was evidence of HCV-RNA in liver explants confirming the theory of $\mathrm{HCV}$ genome compartmentalization in structure derived from endoplasmic reticulum, enabling the virus to avoid clearance from hepatocytes. Besides this plausible explanation, this clinical scenario may deserve further considerations.

Interestingly, a recent study has shown that up to one half of patients treated with DAAs while on a transplant list, and achieving serum HCV-RNA undetectability, display the presence of residual HCV-RNA in their liver explanted [6]. In the same study, however, only patients who had high liver HCV-RNA concentrations experienced virological relapse after LT, while a moderate amount of liver HCV-RNA does not seem to be correlated with HCV recurrence [5].

Similarly, our two patients conceivably did not recur because of their low levels of HCV-RNA in serum and liver at the time of surgery, possibly reflecting a specific viral unfitness to replicate. The latter, in turn, could be the consequence of the selection of RAVs, as demonstrated in case no. 1 , or to other unknown viral-related and/or host-related factors. Whichever the cause, it is remarkable that in both cases, HCV infection did not recurred despite the start and maintenance of immunosuppressive therapy. On the other hand, although recent report suggested a possible role of mTOR in favouring viral decay [7], this issue remains controversial based on a thorough review of the current literature.

We believe that our two cases may add some relevant information to the current controversy about the optimal timing of treatment with DAAs in the liver transplant setting. They show, in fact, that even apparently unsuccessful pre-emptive therapies are capable to avoid HCV recurrence after OLT, hence suggesting that even short or suboptimal courses in some cases can prove to be effective. Indeed, these two cases demonstrate that treatment failure after a course of DAAs in wait-listed patients does not necessarily have negative implications and that may be associated with viral eradication in the long run after early OLT in patients with low HCV-RNA levels in both serum and liver at the time of surgery.

\section{Compliance with ethical standards}

Conflict of interest The authors who have taken part in this study declare that they have nothing to disclose regarding funding or conflict of interest with respect to this manuscript.

\section{References}

1. Forman L, Lucey MR. The association between hepatitis $\mathrm{C}$ infection and survival after orthotopic liver transplantation. Gastroenterology. 2002:889-896. doi:10.1053/gast.2002.32418. 
2. Roche B, Sebagh M, Canfora ML, et al. Hepatitis C virus therapy in liver transplant recipients: response predictors, effect on fibrosis progression, and importance of the initial stage of fibrosis. Liver Transpl. 2008:1766-1777. doi:10.1002/lt.21635.

3. Gane EJ, Agarwal K. Directly acting antivirals (DAAs) for the treatment of chronic hepatitis $\mathrm{C}$ virus infection in liver transplant patients: "a flood of opportunity." Am J Transplant. 2014:994-1002. doi:10.1111/ajt.12714.

4. Garcia-Retortillo M, Forns X, Feliu A, et al. Hepatitis C virus kinetics during and immediately after liver transplantation. Hepatology. 2002;353. doi:10.1053/jhep.2002.31773.
5. Curry MP, Forns X, Chung RT, et al. Sofosbuvir and ribavirin prevent recurrence of $\mathrm{HCV}$ infection after liver transplantation: an open-label study. Gastroenterology.. 2015;148:e1.

6. Gambato M, Pèrez-Del-Pulgar S, Hedskog C, et al. Hepatitis C virus RNA persists in liver explants of most patients awaiting liver transplantation treated with an interferon-free regimen. Gastroenterology. 2016;151:e3.

7. Stöhr S, Costa R, Sandmann L, et al. Host cell mTORC1 is required for HCV RNA replication. Gut. 2016;65:2017-2028. 